\title{
Convergence of a difference method for a system of first order partial differential equations of hyperbolic type
}

\author{
Stefan BaI (Rzeszów)
}

\begin{abstract}
The present paper deals with the quasi-linear system of the differential equations

$$
z_{t}=A(t, x, z) z_{x}+b(t, x, z),
$$

where $A(t, x, z)$ is a matrix with the elements $a_{i j}(t, x, z), i, j=1,2, \ldots, n$ and $b(t, x, z), z(t, x)$ are vector functions and with the corresponding system of difference equations with central differences.

This scheme guarantees better convergence than the schemes with the forward differences and with the backward differences. Assuming that the system has a solution for the given initial conditions and for suitable assumptions, we show the convergence of the method for a suitable quotient $h / k$, where $k, h$ are the lengths of the mesh in the directions of the $t$-axis and the $x$-axis.

The method of the proof consists in taking the difference inequality for the error, i.e. for the difference $u-v$, where $u(t, x)$ is the solution of the system of the differential equations and $v$ is the solution of the system of the difference equations.
\end{abstract}

1. We shall deal with the system of quasi-linear partial differential equations of two independent variables

(1) $z_{t}^{i}=\sum_{j=1}^{n} a_{i j}\left(t, x, z_{1}, \ldots, z_{n}\right) z_{x}^{j}+b_{i}\left(t, x, z_{1}, \ldots, z_{n}\right), \quad i=1,2, \ldots, n$

on the domain $G: 0 \leqslant t \leqslant \alpha,-\alpha \leqslant x \leqslant \alpha,-\infty<z_{i}<+\infty$.

In our earlier paper one of the simplest difference schemes was applied to this system. Now we shall apply another method, giving better convergence. A similar scheme was applied to a special system by Burton Wendroff $\left({ }^{1}\right)$.

System (1) can be written in the form

$$
z_{t}=A(t, x, z) z_{x}+b(t, x, z),
$$

where $A(t, x, z)$ is a matrix of the elementes $a_{i j}(t, x, z), i, j=1,2, \ldots$ $\ldots, n, z(t, x)=\left[z_{1}(t, x), \ldots, z_{n}(t, x)\right]$ is the vector function and $z_{t}, z_{x}$ are its partial derivatives.

(1) Burton Wendroff, Theoretical numerical analysis, New York 1966. 
The difference equation will have the form

$$
\begin{aligned}
& \frac{v^{\mu+1, l}-v^{\mu l}}{k}=A\left(t^{\mu+\downarrow}, x^{l}, v^{\mu+t_{l} l}\right) \frac{v^{\mu, l+1}-v^{\mu l-1}}{2 h}+\frac{k}{2 h} A\left(t^{\mu+\downarrow}, x^{l}, v^{\mu+1, l}\right) \times \\
& \times\left[A\left(t^{\mu}, x^{l+\ddagger}, v^{\mu, l+\downarrow}\right) \frac{v^{\mu, l+1}-v^{\mu l}}{h}-A\left(t^{\mu}, x^{l-\ddagger}, v^{\mu l-t}\right) \frac{v^{\mu l}-v^{\mu l-1}}{h}\right]+ \\
& +\frac{k}{2 h} A\left(t^{\mu+t}, x^{l}, v^{\mu+t, l}\right)\left[b\left(t^{\mu}, x^{l+1}, v^{\mu, l+t}\right)-b\left(t^{\mu}, x^{l-\frac{t}{t}}, v^{\mu, l-\frac{1}{t}}\right)\right]+ \\
& +\quad+b\left(t^{\mu+1}, x^{l}, v^{\mu+t, l}\right), \\
& \text { where } v^{\mu l}=\left(v_{1}^{\mu l}, \ldots, v_{n}^{\mu l}\right)=\left(v_{1}\left(t^{\mu}, x^{l}\right), \ldots, v_{n}\left(t^{\mu}, x^{l}\right)\right)
\end{aligned}
$$

is a value-estimating solution at the nodal point of the net $\left(t^{\mu}, x^{l}\right)$, $t^{\mu}=\mu k, \mu=0,1,2, \ldots, m_{1}, x^{l}=l h, l=-m_{2},-m_{2}+1, \ldots,-2,-1$, $0,1,2, \ldots, m_{2}$, where $k=a / m_{1}$ denotes the length of the mesh in the direction of the $t$-axis, and $h=\alpha / m_{2}$ denotes the length of the mesh in the direction of the $x$-axis.

We shall denote the vectors in the $m$-dimensional space by the small letters $v=\left(v_{1}, \ldots, v_{n}\right)$, and the matrices with the capital letters. $A=\left[a_{i j}\right]$ is the matrix of the elements $a_{i j}, i, j=1,2, \ldots, n$.

By $I$ we shall denote the identity matrix.

We introduce the definitions of the norm of the matrix and of the vector in the $n$-dimensional space $\|A\|=n \max \left|a_{i j}\right|,\|a\|=\sqrt{a_{1}^{2}+\ldots+a_{n}^{2}}$. The definitions imply the inequalities

$$
\begin{aligned}
& \|a+b\| \leqslant\|a\|+\|b\|, \quad\|A+B\| \leqslant\|A\|+\|B\|, \quad\|A B\| \leqslant\|A\|\|B\| \\
& \text { and }\|A x\| \leqslant\|A\|\|x\| \text {. }
\end{aligned}
$$

We shall consider the set $\Omega$ of the vector functions $u\left(x^{l}\right)$ such that $u\left(x^{l}\right)=0$ for sufficiently large $\left|x^{l}\right|$.

If $u$ and $v$ are the vector functions, then we denote the scalar product by $u v$ and we define:

$$
u v=\sum_{l=-\infty}^{\infty} u\left(x^{l}\right) v\left(x^{l}\right) .
$$

We shall need the theorem on difference inequalities.

THEOREM. If the sequence $s^{\mu}, \mu=0,1,2, \ldots, n$ satisfies the inequality

$$
\frac{s^{\mu+1}-s^{\mu}}{k} \leqslant L\left(s^{\mu}+h^{3 / 2}\right) / h^{3 / 2}
$$

and the initial condition $s^{0}=0$, where $L$ is the non-negative constant, then

$$
s^{\mu} \leqslant L \mu k h^{3 / 2} /(1-L \mu \hbar)
$$


2. Assumptions $Z$.

$1^{\circ} a_{i j}\left(t, x, z_{1}, \ldots, z_{n}\right)$ and $b_{i}\left(t, x, z_{1}, \ldots, z_{n}\right)$ are of class $C^{1}$ on $G$ and are bounded together with the derivatives of first order on $G$,

$$
\begin{gathered}
\left|a_{i j}\left(t, x, z_{1}, \ldots, z_{n}\right)\right| \leqslant \frac{M}{n}, \quad\left|\frac{\partial a_{i j}}{\partial t}\right|, \quad\left|\frac{\partial a_{i j}}{\partial x}\right| \leqslant \frac{M}{n}, \quad\left|\frac{\partial a_{i j}}{\partial z_{i}}\right| \leqslant \frac{M}{n}, \\
\left|b_{i}\right| \leqslant M,\left|\frac{\partial b_{i}}{\partial z_{k}}\right| \leqslant \frac{M}{n} .
\end{gathered}
$$

$2^{\circ}$ The function $u(t, x)=\left[u_{1}(t, x), \ldots, u_{n}(t, x)\right]$ of class $C^{2}$ on a rectangle $0 \leqslant t \leqslant a,-\alpha \leqslant x \leqslant \alpha$ is a solution of system (1) satisfying the initial conditions

$$
u(0, x)=\varphi(x) \quad \text { for } \quad-a \leqslant x \leqslant a .
$$

On this rectangle the inequalities $\|u\|,\left\|u_{t}\right\|,\left\|u_{x}\right\|,\left\|u_{t x}\right\|,\left\|u_{x x}\right\|,\left\|u_{t t}\right\| \leqslant c$, are satisfied.

$3^{\circ}$ System (1) is assumed to be strongly hyperbolic in $G$, that is, all eigenvalues $\lambda_{i}=\lambda_{i}\left(t, x, z_{1}, \ldots, z_{n}\right), i=1,2, \ldots, n$ of the matrix $A\left(t, x, z_{1}, \ldots, z_{n}\right)=\left[a_{i j}\left(t, x, z_{1}, \ldots, z_{n}\right)\right]$ are real, $\lambda_{i} \neq \lambda_{k}$ for $i \neq k$ and $\lambda_{i}<0$ for $i=1,2, \ldots, n$.

By $P$ we shall denote the matrix the columns of which are the eigenvectors $p^{i}$, corresponding to the values $\lambda_{i}$ of matrix $A$.

We have $\left(A-\lambda_{i} I\right) p^{i}=0$.

It follows from the assumptions that $p^{i}$ are linearly independent, $P A P^{-1}=\Lambda$, where

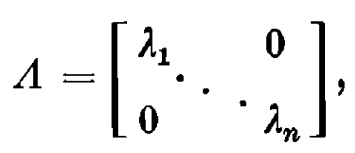

$p_{i j}$ - the elements of matrix $P$ are the analytic functions of elements $a^{i j}$.

Under these assumptions the elements $p_{i j}$ of the matrix $P$ transforming the matrix $A=\left[a_{i j}\left(t, x, u_{1}(t, x), \ldots, u_{n}(t, x)\right)\right]$ are functions of class $C^{1}$ of two variables $(t, x)$.

From the boundary of the functions $a_{i j}$ on $G$ and from the assumptions of concerning $\lambda_{i}$ it follows that there is a positive constant $H$ such that $\|P\|,\left\|P^{-1}\right\|,\left\|P_{t}\right\|,\left\|P_{x}\right\|,\left\|P_{t}^{-1}\right\|,\left\|P_{x}^{-1}\right\|,\left\|\Lambda_{t}\right\|,\left\|\Lambda_{x}\right\|,\|\Lambda\| \leqslant H$ on $G$, where $P$ is matrix of elements $P_{t}=\left[p_{t}^{i j}(t, x)\right]$.

We suppose that the vectors $v^{\mu l}$ satisfy the following initial conditions:

$$
v^{0 l}=\varphi\left(x^{l}\right)
$$

Further $v^{\mu l}$ will be taken from difference equation (2). 
We write:

$$
L=R+R_{1}, \quad R=2 H+12 H^{2}+112 H^{3}, \quad R_{1}=\max \left[a_{1}, a_{2}\right],
$$

where

$$
\begin{aligned}
a_{1}= & 2 H^{2}+4 n H^{3} M^{2}+4 H^{2} M^{3}+\left(2 n H^{2} M^{2}+4 H^{2} M^{3}\right)(H+3 H M+n c H M)^{2}, \\
a_{2}= & H^{2}+13 H^{2} M+17 H^{2} M^{2}+H^{3} M+3 H^{3} M^{2}+n c H^{3} M^{2}+ \\
& +2 n c H^{2} M+2 n H^{3} M^{2}+6 n H^{3} M^{3}+2 n^{2} c H^{3} M^{3}+8 n c H^{2} M^{2}+ \\
& +8 H^{3} M^{3}+24 H^{3} M^{4}+8 n c H^{3} M^{4}+5 n^{2} c H^{2} M+9 n^{2} c H^{2} M^{2}+ \\
& +2 n^{3} c H^{2} M^{2}+n^{3} c^{2} H^{2} M^{2}+2 n H^{2} M^{2}+4 n H^{2} M^{3}+2 n^{2} c H^{2} M^{3} .
\end{aligned}
$$

THEOREM 1. We suppose that Assumtions $\mathrm{Z}$ are satisfied and the values $u^{\mu l}, v^{\mu l}$ are defined by (1), (2), (3), (4).

We assume $h / k$ constant such that $\lambda_{i}+h / k>0, i=1,2, \ldots, n$, where $\lambda_{i}$ denote characteristic values of matrix $A=\left[a_{i j}(t, x, z)\right]$.

Then $\left\|u^{\mu l}-v^{\mu l}\right\| \leqslant H L \mu k h^{3 / 2} /(1-L \mu k)$.

This inequality implies the convergence of the method:

$$
\left\|u^{\mu l}-v^{\mu l}\right\| \rightarrow 0, \quad \text { where } h, k \rightarrow 0 .
$$

Now we shall show three lemmas.

We shall shortend the notation:

$$
\begin{gathered}
A\left(t^{\mu}, x^{l}, u^{\mu l}\right)=A^{(u) \mu l}, \quad A\left(t^{\mu}, x^{l}, v^{\mu l}\right)=A^{\mu l}, \quad b\left(t^{\mu}, x^{l}, u^{\mu l}\right)=b^{(u) \mu_{l}}, \\
b\left(t^{\mu}, x^{l}, v^{\mu l}\right)=b^{\mu l}, \quad D u^{\mu l}=\frac{u^{\mu+1, l}-u^{\mu l}}{k}, \quad D^{+} u^{\mu l}=\frac{u^{\mu l+1}-u^{\mu l}}{h}, \\
D^{-} u^{\mu l}=\frac{u^{\mu l}-u^{\mu l-1}}{h}, \quad D^{1} u^{\mu l}=\frac{u^{\mu l+1}-u^{\mu l-1}}{2 h}, \\
D^{2} u^{\mu l}=\frac{u^{\mu l+1}-2 u^{\mu l}+u^{\mu l-1}}{2 h^{2}},
\end{gathered}
$$

$2^{\mathrm{o}} \quad w_{1}^{\mu l}=\left[A^{(u) \mu+1 / 2, l} A^{(u) \mu l+1 / 2}-A^{\mu+1 / 2, l} A^{\mu l+1 / 2}\right] D^{+} u^{\mu l}-$

$$
\begin{gathered}
-\left[A^{(u) \mu+1 / 2, l} A^{(u) \mu l-1,2}-A^{\mu+1 / 2, l} A^{\mu l-1 / 2}\right] D^{-} u^{\mu l}, \\
w_{2}^{\mu l}=\left[A^{(u) \mu+1 / 2, l}-A^{\mu+1 / 2, l}\right] D^{1} u^{\mu l}, \\
w_{3}^{\mu l}=A^{(u) \mu+1 / 2, l} b^{(u) \mu l+1 / 2}-A^{\mu+1 / 2, l} b^{\mu l+1 / 2}-A^{(u) \mu+1 / 2, l} b^{(u) \mu l-1 / 2}+ \\
+A^{\mu+1 / 2, l} b^{\mu l-1 / 2},
\end{gathered}
$$

$3^{\circ}$

$$
\begin{aligned}
& B_{1}^{\mu l}=\frac{1}{2 h}\left[A^{\mu+1 / 2, l}-A^{(u) \mu l}\right] \\
& B_{2}^{\mu l}=\frac{1}{h}\left[A^{\mu+1 / 2, l} A^{\mu l+1 / 2}-A^{(u) \mu l} A^{(u) \mu l}\right], \\
& B_{3}^{\mu l}=\frac{1}{h}\left[A^{\mu+1, ?, l} A^{\mu l-1 / 2}-A^{(u) \mu l} A^{(u) \mu l}\right] .
\end{aligned}
$$


LEMMA 1. Vectors $r^{\mu l}=u^{\mu l}-v^{\mu l}$ satisfy the difference equation

$$
\begin{aligned}
D r^{\mu l}= & A^{(u) \mu l} D^{1} r^{\mu l}+k\left(A^{(u) \mu l}\right)^{2} D^{2} r^{\mu l}+\left(B_{1}^{\mu l}+\frac{k}{2 h} B_{2}^{\mu l}\right) r^{\mu, l+1}- \\
& -\frac{k}{2 h}\left(B_{2}^{\mu l}+B_{3}^{\mu l}\right) r^{\mu l}+\left(\frac{k}{2 h} B_{3}^{\mu l}-B_{1}^{\mu l}\right) r^{\mu l-1}+\frac{k}{2 h} w_{1}^{\mu l}+w_{2}^{\mu l}+\frac{k}{2 h} w_{3}^{\mu l}+ \\
& +B_{u}^{\mu+1 / 2, l} r^{\mu+1 / 2, l}+\eta^{\mu+1 / 2, l}
\end{aligned}
$$

Proof. Vectors $u\left(t^{\mu}, x^{l}\right)=u^{\mu l}(u(t, x)$ - the solution of differential equation (1)) satisfy the system of equations

(5) $\frac{u^{\mu+1, l}-u^{\mu l}}{k}$

$$
\begin{aligned}
= & A\left(t^{\mu+1 / 2}, x^{l}, u^{\mu+1 / 2, l}\right) \frac{u^{\mu l+1}-u^{\mu l-1}}{2 h}+\frac{k}{2 h} A\left(t^{\mu+1 / 2}, x^{l}, u^{\mu+1 / 2, l}\right) \times \\
& \times\left[A\left(t^{\mu}, x^{l+1 / 2}, u^{\mu l+1 / 2}\right) \frac{u^{\mu l+1}-u^{\mu l}}{h}-A\left(t^{\mu}, x^{l-1 / 2}, u^{\mu l-1 / 2}\right) \frac{u^{\mu l}-u^{\mu l-1}}{h}\right]+ \\
& +\frac{k}{2 h} A\left(t^{\mu+1 / 2}, x^{l}, u^{\mu+1 / 2, l}\right)\left[b\left(t^{\mu}, x^{l+1 / 2}, u^{\mu l+1 / 2}\right)-b\left(t^{\mu}, x^{l-1 / 2}, u^{\mu l-1 / 2}\right)\right]+ \\
& +b\left(t^{\mu+1 / 2}, x^{l}, u^{\mu+1 / 2, l}\right)+\eta^{\mu+1 / 2, l},
\end{aligned}
$$

$\eta^{\mu+1 / 2, l}$ is of order $O\left(h^{2}\right)$.

Let us take a difference $r^{\mu l}=u^{\mu l}-v^{\mu l}$. Then we have

$$
\begin{aligned}
D r^{\mu l}= & D u^{\mu l}-D v^{\mu l}=A^{(u) \mu+1 / 2, l} D^{1} u^{\mu l}-A^{\mu+1 / 2, l} D^{1} v^{\mu l}+ \\
& +\frac{k}{2 h} A^{(u) \mu+1 / 2, l}\left[A^{(u) \mu l+1 / 2} D^{+} u^{\mu l}-A^{(u) \mu l-1 / 2} D^{-} u^{\mu l}\right]- \\
& -\frac{k}{2 h} A^{\mu+1 / 2, l}\left[A^{(u) \mu l+1 / 2} D^{+} v^{\mu l}-A^{\mu l-1 / 2} D^{-} v^{\mu l}\right]+ \\
& +\frac{k}{2 h} A^{(u) \mu+1 / 2, l}\left[b^{(u) \mu l+1 / 2}-b^{\mu l-1 / 2}\right]-\frac{k}{2 h} A^{\mu+1 / 2, l}\left[b^{\mu l+1 / 2}-b^{\mu l-1 / 2}\right]+ \\
& +b^{(u) \mu+1 / 2, l}-b^{\mu+1 / 2, l}+\eta^{\mu+1 / 2, l} .
\end{aligned}
$$

Hence we obtain

(6) $\quad D r^{\mu l}=A^{\mu+1 / 2 l} D^{1} r^{\mu l}+\left[A^{(u) \mu+1 / 2, l}-A^{\mu+1 / 2, l}\right] D^{1} u^{\mu l}+$

$$
+\frac{k}{2 h}\left\{A^{(u) \mu+1 / 2, l} A^{(u) \mu l+1 / 2} D^{+} u^{\mu l}-A^{(u) \mu+1 / 2, l} A^{(u) \mu l-1 / 2} D^{-} u^{\mu l}-\right.
$$




$$
\begin{aligned}
& -A^{\mu+1 / 2, l} A^{\mu l+1 / 2} D^{+} v^{\mu l}+ \\
& \left.+A^{\mu+1 / 2, l} A^{\mu l-1 / 2} D^{-} v^{\mu l}\right\}+\frac{k}{2 h}\left\{A^{(u) \mu+1 / 2, l} b^{(u) \mu l+1 / 2}-\right. \\
& \left.-A^{(u) \mu+1 / 2, l} b^{(u) \mu, l-1 / 2}-A^{\mu+1 / 2, l} b^{\mu l+1 / 2}+A^{\mu+1 / 2, l} b^{\mu l-1 / 2}\right\}+ \\
& +b^{(u) \mu+1 / 2, l}-b^{\mu+1 / 2, l}+\eta^{\mu+1 / 2, l} .
\end{aligned}
$$

In virtue of the equality

$$
\begin{aligned}
\frac{k}{2 h} & \left\{A^{(u) \mu+1 / 2, l} A^{(u) \mu l+1 / 2} D^{+} u^{\mu l}-A^{(u) \mu+1 / 2, l} A^{(u) \mu l-1 / 2} D^{-} u^{\mu l}-\right. \\
& \left.-A^{\mu+1 / 2, l} A^{\mu l+1 / 2} D^{+} v^{\mu l}+A^{\mu+1 / 2, l} A^{\mu l-1 / 2} D^{-} v^{\mu l}\right\} \\
= & \frac{k}{2 h}\left\{A^{\mu+1 / 2, l} A^{\mu l+1 / 2} D^{+} r^{\mu l}+\left[A^{(u) \mu+1 / 2, l} A^{(u) \mu l+1 / 2}-A^{\mu+1 / 2, l} A^{\mu l+1 / 2}\right] D^{+} u^{\mu l}-\right. \\
& \left.-A^{\mu+1 / 2, l} A^{\mu l-1 / 2} D^{-} r^{\mu l}-\left[A^{(u) \mu+1 / 2, l} A^{(u) \mu l-1 / 2}-A^{\mu+1 / 2, l} A^{\mu l-1 / 2}\right] D^{-} u^{\mu l}\right\}
\end{aligned}
$$

we obtain from (6)

$$
\begin{aligned}
& \text { (7) } \quad D r^{\mu l}=A^{\mu+1 / 2, l} D^{1} r^{\mu l}+\frac{k}{2 h}\left[A^{\mu+1 / 2, l} A^{\mu l+1 / 2} D^{+} r^{\mu l}-\right. \\
& \left.-A^{\mu+1 / 2, l} A^{\mu l-1 / 2} D^{-} r^{\mu l}\right]+\frac{k}{2 h} w_{1}^{\mu l}+w_{2}^{\mu l}+\frac{k}{2 h} w_{3}^{\mu l}+B_{u}^{\mu+1 / 2, l} r^{\mu+1 / 2, l}+\eta^{\mu+1 / 2, l}
\end{aligned}
$$

$B_{u}^{\mu+1 / 2, l}$ is the matrix of elements $b_{u j}^{i}\left(t^{\mu+1 / 2}, x^{l}, u^{\mu+1 / 2, l}-\theta r^{\mu+1 / 2, l}\right), 0<\theta<1$. Using the equalities

(9) $\frac{k}{2 h}\left[A^{\mu+1 / 2, l} A^{\mu l+1 / 2} D^{+} r^{\mu l}-A^{\mu+1 / 2, l} A^{\mu l-1 / 2} D^{-} r^{\mu l}\right]$

$$
=k\left(A^{(\mu) \mu l}\right)^{2} D^{2} r^{\mu l}+\frac{k}{2 h} B_{2}^{\mu l}\left(r^{\mu l+1}-r^{\mu l}\right)-\frac{k}{2 h} B_{3}^{\mu l}\left(r^{\mu l}-r^{\mu l-1}\right),
$$

we obtain the equality

$$
\begin{aligned}
D r^{\mu l} & =A^{(u) \mu l} D^{1} r^{\mu l}+k\left(A^{(u) \mu l}\right)^{2} D^{2} r^{\mu l}+B_{1}^{\mu l}\left(r^{\mu l+1}-r^{\mu l-1}\right)+\frac{k}{2 h} B_{2}^{\mu l}\left(r^{\mu l+1}-r^{\mu l}\right)- \\
& -\frac{k}{2 h} B_{3}^{\mu l}\left(r^{\mu l}-r^{\mu l-1}\right)+\frac{k}{2 h} w_{1}^{\mu l}+w_{2}^{\mu l}+\frac{k}{2 h} w_{3}^{\mu l}+B_{u}^{\mu+1 / 2, l} r^{\mu+1 / 2, l}+\eta^{\mu+1 / 2, l}
\end{aligned}
$$


After a suitable ordering of terms we have

$$
\begin{aligned}
& D r^{\mu l}= A^{(u) \mu l} D^{1} r^{\mu l}+k\left(A^{(u) \mu l}\right)^{2} D^{2} r^{\mu l}+\left(B_{1}^{\mu l}+\frac{k}{2 h} B_{2}^{\mu l}\right) r^{\mu l+1}- \\
&-\frac{k}{2 h}\left(B_{2}^{\mu l}+B_{3}^{\mu l}\right) r^{\mu l}+\left(\frac{k}{2 h} B_{3}^{\mu l}-B_{1}^{\mu l}\right) r^{\mu l-1}+\frac{k}{2 h} w_{1}^{\mu l}+w_{2}^{\mu l}+\frac{k}{2 h} w_{3}^{\mu l}+ \\
&+B_{u}^{\mu+1 / 2, l} r^{\mu+1 / 2, l}+\eta^{\mu+1 / 2, l}
\end{aligned}
$$

The proof of Lemma 1 is ended.

Let us use the transformation $\varrho^{\mu l}=P^{\mu l} r^{\mu l}$ and the inverse transformation $r^{\mu l}=P^{-1 \mu l} \varrho^{\mu l}$, where $P^{\mu l} A^{(u) \mu l} P^{-1 \mu l}=\Lambda^{\mu l}$.

Let us introduce the following notations:

$$
\begin{array}{r}
E^{\mu l+1}=\frac{k}{2 h} P_{t}^{\mu l} A^{(u) \mu l} P^{-1 \mu l}+\frac{1}{2} P^{\mu l} A^{(u) \mu l} P_{x}^{-1 \mu l}+\frac{k}{2} P_{t}^{\mu l} A^{(u) \mu l} P_{x}^{-1 \mu l}+ \\
+\frac{k^{2}}{2 h^{2}} P_{l}^{\mu l}\left(A^{(u) \mu l}\right)^{2} P^{-1 \mu l}+\frac{k}{2 h} P^{\mu+1, l}\left(A^{(u) \mu l}\right)^{2} P^{-1 \mu l} \\
E^{\mu l}=-\frac{k^{2}}{h^{2}} P_{t}^{\mu l}\left(A^{(u) \mu l}\right)^{2} P^{-1 \mu l}-\frac{k}{2 h} P^{\mu+1, l}\left(A^{(u) \mu l}\right)^{2} P_{x}^{-1 \mu l}+ \\
+P^{\mu+1, l}\left(A^{(u) \mu l}\right)^{2} P_{x}^{-1 \mu l}+P_{t}^{\mu l} P^{-1 \mu l}
\end{array}
$$

$$
\begin{aligned}
E^{\mu l-1} & =-\frac{k}{2 h} P_{t}^{\mu l} A^{(u) \mu l} P^{-1 \mu l}-\frac{1}{2} P^{\mu l} A^{(u) \mu l} P_{x}^{-1 \mu l}-\frac{k}{2} P_{t}^{\mu l} A^{(u) \mu l} P_{x}^{-1 \mu l}+ \\
& +\frac{k^{2}}{2 h^{2}} P_{t}^{\mu l}\left(A^{(u) \mu l}\right)^{2} P^{-1 \mu l}-\frac{k}{2 h} P^{\mu+1, l}\left(A^{(u) \mu l}\right)^{2} P_{x}^{-1 \mu l-1}+P^{\mu+1, l} A^{(u) \mu l} P_{x}^{-1 \mu l}
\end{aligned}
$$

$2^{\circ}$

$$
S_{\mu} \varrho^{\mu l}=\varrho^{\mu l}+k \Lambda^{\mu l} D^{1} \varrho^{\mu l}+k^{2}\left(\Lambda^{\mu l}\right)^{2} D^{2} \varrho^{\mu l}
$$

$$
\begin{gathered}
G_{\mu} \varrho^{\mu l}=E^{\mu l+1} \varrho^{\mu l+1}+E^{\mu l} \varrho^{\mu l}+E^{\mu l-1} \varrho^{\mu l-1}+P^{\mu+1, l} B_{u}^{\mu+1 / 2, l} P^{-1 \mu+1 / 2, l} \varrho^{\mu+1 / 2, l}+ \\
+P^{\mu+1, l}\left(B_{1}^{\mu l}+\frac{k}{2 h} B_{2}^{\mu l}\right) P^{-1 \mu l+1} \varrho^{\mu l+1}-\frac{k}{2 h} P^{\mu+1, l}\left(B_{2}^{\mu l}+B_{3}^{\mu l}\right) P^{-1 \mu l} \varrho^{\mu l}+ \\
+P^{\mu+1, l}\left(\frac{k}{2 h} B_{3}^{\mu l}-B_{1}^{\mu l}\right) P^{-1 \mu l-1} \varrho^{\mu l-1}+\frac{k}{2 h} P^{\mu+1, l} w_{1}^{\mu l}+ \\
+P^{\mu+1, l} w_{2}^{\mu l}+\frac{k}{2 h} P^{\mu+1, l} w_{3}^{\mu l},
\end{gathered}
$$

$3^{\circ}$

$$
\varepsilon^{\mu l}=P^{\mu+1, l} \eta^{\mu+1 / 2, l} .
$$

For $\varrho^{\mu l}$ we shall find the difference equation. 
LENMA 2. Vectors $\varrho^{\mu l}=P^{\mu l} r^{\mu l}$ satisfy the difference equation

$$
\varrho^{\mu+1, l}=S_{\mu} \varrho^{\mu l}+k G_{\mu} \varrho^{\mu l}+k \varepsilon^{\mu l} .
$$

Proof.

$$
\begin{aligned}
& D \varrho^{\mu l}=D\left(P^{\mu l} r^{\mu l}\right)=P^{\mu+1, l} D \varrho^{\mu l}+\frac{1}{k}\left(P^{\mu+1, l}-P^{\mu l}\right) r^{\mu l}=P^{\mu+1, l} A^{(u) \mu l} D^{1} r^{\mu l}+ \\
+ & k P^{\mu+1, l}\left(A^{(u) \mu l}\right)^{2} D^{2} r^{\mu l}+P^{\mu+1, l}\left[\left(B_{1}^{\mu l}+\frac{k}{2 h} B_{2}^{\mu l}\right) r^{\mu l+1}-\frac{k}{2 h}\left(B_{2}^{\mu l}+B_{3}^{\mu l}\right) r^{\mu l}+\right. \\
+ & \left.\left(\frac{k}{2 h} B_{3}^{\mu l}-B_{1}^{\mu l}\right) r^{\mu, l-1}+\frac{k}{2 h} w_{1}^{\mu l}+w_{2}^{\mu l}+\frac{k}{2 h} w_{3}^{\mu l}+B_{u}^{\mu+1 / 2, l} r^{\mu+1 / 2, l}+\eta^{\mu+1 / 2, l}\right]+ \\
+ & P_{t}^{\mu l} r^{\mu l}, \quad \text { where } P_{t}^{\mu l}=\frac{1}{k}\left(P^{\mu+1, l}-P^{\mu l}\right) .
\end{aligned}
$$

Applying the inverse transformation, we have

$$
\begin{aligned}
& D \varrho^{\mu l}=P^{\mu+1, l} A^{(u) \mu l} D^{1}\left(P^{-1 \mu l} \varrho^{\mu l}\right)+k P^{\mu+1, l}\left(A^{(u) \mu l}\right)^{2} D^{2}\left(P^{-1 \mu l} \varrho^{\mu l}\right)+ \\
& \quad+P^{\mu+1, l}\left[\left(B_{1}^{\mu l}+\frac{k}{2 h} B_{2}^{\mu l}\right) P^{-1 \mu l+1} \varrho^{\mu l+1}-\frac{k}{2 h}\left(B_{2}^{\mu l}+B_{3}^{\mu l}\right) P^{-1 \mu l} \varrho^{\mu l}+\right. \\
& \quad+\left(\frac{k}{2 h} B_{3}^{\mu l}-B_{1}^{\mu l}\right) P^{-1 \mu l-1} \varrho^{\mu l-1}+\frac{k}{2 h} w_{1}^{\mu l}+w_{2}^{\mu l}+\frac{k}{2 h} w_{3}^{\mu l}+ \\
& \left.\quad+B_{u}^{\mu+1 / 2, l} P^{-1 \mu+1 / 2, l} \varrho^{\mu+1 / 2, l}+\eta^{\mu+1 / 2, l}\right]+P_{l}^{\mu l} P^{-1 \mu l} \varrho^{\mu l}
\end{aligned}
$$

Now we shall transform the first two terms.

Writing

$$
J=P^{\mu+1, l} A^{(u) \mu l} D^{1}\left(P^{-1 \mu l} \varrho^{\mu l}\right)+k P^{\mu+1, l}\left(A^{(u) \mu l}\right)^{2} D^{2}\left(P^{-1 \mu l} \varrho^{\mu l}\right),
$$

we have

$$
\begin{aligned}
J= & P^{\mu+1, l} A^{(u) \mu l} D^{1}\left(P^{-1 \mu l} \varrho^{\mu l}\right)+k P^{\mu+1, l}\left(A^{(u) \mu l}\right)^{2} D^{2}\left(P^{-1 \mu l} \varrho^{\mu l}\right) \\
= & P^{\mu+1, l} A^{(u) \mu l} P^{-1 \mu l+1} D^{1} \varrho^{\mu l}+\frac{1}{2 h} P^{\mu+1, l} A^{(u) \mu l}\left(P^{-1 \mu l+1}-P^{-1 \mu l-1}\right) \varrho^{\mu l-1}+ \\
& +k P^{\mu+1, l}\left(A^{(u) \mu l}\right)^{2}\left[P^{-1 \mu, l-1} \frac{1}{2 h} D^{+} \varrho^{\mu l}+\frac{1}{2 h^{2}}\left(P^{-1 \mu l+1}-P^{-1 \mu l}\right) \varrho^{\mu l}--\right. \\
& \left.-\frac{1}{2 h} P^{-1 \mu l} D^{-} \varrho^{\mu l}-\frac{1}{2 h^{2}}\left(P^{-1 \mu l}-P^{-1 \mu l-1}\right) \varrho^{\mu l-1}\right] .
\end{aligned}
$$

Since

$$
\frac{1}{2 h}\left(D^{+} \varrho^{\mu l}-D^{-} \varrho^{\mu l}\right)=D^{2} \varrho^{\mu l} \quad \text { and } \quad P^{-1 \mu l}-P^{-1 \mu l-1}=h P_{x}^{-1 \mu l-1}
$$


we obtain

$$
\begin{aligned}
J= & P^{\mu+1, l} A^{(u) \mu l} P^{-1 \mu l+1} D^{1} \varrho^{\mu l}+k P^{\mu+1, l}\left(A^{(u) \mu l}\right)^{2}\left[\frac{1}{2 h} P^{-1 \mu l} D^{+} \varrho^{\mu l}+\right. \\
& +\frac{1}{2} P_{x}^{-1 \mu l} D^{+} \varrho^{\mu l}-\frac{1}{2 h} P^{-1 \mu l} D^{-} \varrho^{\mu l}+\frac{1}{2 h^{2}}\left(P^{-1 \mu l+1}-P^{-1 \mu l}\right) \varrho^{\mu l}- \\
& \left.-\frac{1}{2 h^{2}}\left(P^{-1 \mu l}-P^{-1 \mu l-1}\right) \varrho^{\mu l-1}\right]+\frac{1}{2 h} P^{\mu+1, l} A^{(u) \mu l}\left(P^{-1 \mu, l+1}-P^{-1 \mu l-1}\right) \varrho^{\mu l-1} \\
= & P^{\mu+1, l} A^{(u) \mu l} P^{-1 \mu l+1} D^{1} \varrho^{\mu l}+k P^{\mu+1, l}\left(A^{(u) \mu l}\right)^{2} P^{-1 \mu l} D^{2} \varrho^{\mu l}+ \\
& +\frac{k}{2 h}\left[P^{\mu+1, l}\left(A^{(u) \mu l}\right)^{2} P_{x}^{-1 \mu l}\left(\varrho^{\mu l+1}-\varrho^{\mu l}\right)+P^{\mu+1, l}\left(A^{(u) \mu l}\right)^{2} P_{x}^{-1 \mu l} \varrho^{\mu l}-\right. \\
& \left.-P^{\mu+1, l}\left(A^{(u) \mu l}\right)^{2} P_{x}^{-1 \mu l-1} \varrho^{\mu l-1}\right]+P^{\mu+1, l} A^{(u) \mu l} P_{x}^{-1 \mu l} \varrho^{\mu l-1} .
\end{aligned}
$$

Since $P^{\mu+1, l}=P^{\mu l}+k P_{t}^{\mu l}$, it follows that

$$
\begin{aligned}
& J=\left(P^{\mu l}+k P_{t}^{\mu l}\right) A^{(u) \mu l}\left(P^{-1 \mu l}+h P_{x}^{-1 \mu l}\right) D^{1} \varrho^{\mu l}+ \\
& +k\left(P^{\mu l}+k P_{l}^{\mu l}\right)\left(A^{(u) \mu l}\right)^{2} P^{-1 \mu l} D^{2} \varrho^{\mu l}+\frac{k}{2 h}\left[P^{\mu+1, l}\left(A^{(u) \mu l}\right)^{2} P_{x}^{-1 \mu l}\left(\varrho^{\mu, l+1}-\varrho^{\mu l}\right)+\right. \\
& \left.+P^{\mu+1, l}\left(A^{(u) \mu l}\right)^{2} P_{x}^{-1 \mu l} \varrho^{\mu l}-P^{\mu+1, l}\left(A^{(u) \mu l}\right)^{2} P_{x}^{-1 \mu l-1} \varrho^{\mu l-1}\right]+ \\
& +P^{\mu+1, l} A^{(u) \mu l} P_{x}^{-1 \mu l} \varrho^{\mu l-1}
\end{aligned}
$$

We know that $P^{\mu l} A^{(u) \mu l} P^{-1 \mu l}=\Lambda^{\mu l}$; therefore we obtain

$$
J=\Lambda^{\mu l} D^{1} \varrho^{\mu l}+k\left(\Lambda^{\mu l}\right)^{2} D^{2} \varrho^{\mu l}+\left[\frac{k}{2 h} P_{t}^{\mu l} A^{(u) \mu l} P^{-1 \mu l}+\frac{\dot{1}}{2} P^{\mu l} A^{(u) \mu l} P_{x}^{-1 \mu l}+\right.
$$$$
+\frac{k}{2} P_{t}^{\mu l} A^{(u) \mu l} P_{x}^{-1 \mu l}+\frac{k^{2}}{2 h^{2}} P_{t}^{\mu l}\left(A^{(u) \mu l}\right)^{2} P^{-1 \mu l}+\frac{k}{2 h} P^{\mu+1, l}\left(A^{(u) \mu l}\right)^{2} P^{-1 \mu l} \varrho^{\mu l+1}+
$$$$
+\left[-\frac{k^{2}}{h^{2}} P_{l}^{\mu l}\left(A^{(u) \mu l}\right)^{2} P^{-1 \mu l}-\frac{k}{2 h} P^{\mu+1, l}\left(A^{(u) \mu l}\right)^{2} P_{x}^{-1 \mu l}+P^{\mu+1, l}\left(A^{(u) \mu l}\right)^{2} P_{x}^{-1 \mu l}\right] \varrho^{\mu l}+
$$$$
+\left[-\frac{k}{2 h} P_{t}^{\mu l}\left(A^{(u) \mu l}\right)^{2} P^{-1 \mu l}-\frac{1}{2} P^{\mu l} A^{(u) \mu l} P_{x}^{-1 \mu l}-\frac{k}{2} P_{t}^{\mu l} A^{(u) \mu l} P_{x}^{-1 \mu l}+\right.
$$$$
\left.+\frac{k^{2}}{2 h^{2}} P_{t}^{\mu l}\left(A^{(u) \mu l}\right)^{2} P^{-1 \mu l}-\frac{k}{2 h} P^{\mu+1, l}\left(A^{(u) \mu l}\right)^{2} P_{x}^{-1 \mu l-1}+P^{\mu+1, l} A^{(u) \mu l} P_{x}^{-1 \mu l}\right] \varrho^{\mu l-1}
$$ 
Using this equality and (10) and notations $1^{\circ}$ we have

$$
\begin{aligned}
D \varrho^{\mu l}= & A^{\mu l} D^{1} \varrho^{\mu l}+k\left(\Lambda^{\mu l}\right)^{2} D^{2} \varrho^{\mu l}+E^{\mu l+1} \varrho^{\mu l+1}+E^{\mu l} \varrho^{\mu l}+ \\
& +E^{\mu l-1} \varrho^{\mu l-1}+P^{\mu+1, l} B_{u}^{\mu+1 / 2, l} P^{-1 \mu+1 / 2, l} \varrho^{\mu+1 / 2, l}+\frac{k}{2 h} P^{\mu+1, l} w_{1}^{\mu l}+ \\
& +P^{\mu+1, l} w_{2}^{\mu l}+\frac{k}{2 h} P^{\mu+1, l} w_{3}^{\mu l}+P^{\mu+1, l} \eta^{\mu+1 / 2, l}+ \\
& +P^{\mu+1, l}\left(B_{1}^{\mu l}+\frac{k}{2 h} B_{2}^{\mu l}\right) P^{-1 \mu l+1} \varrho^{\mu l+1}- \\
& -\frac{k}{2 h} P^{\mu+1, l}\left(B_{2}^{\mu l}+B_{3}^{\mu l}\right) P^{-1 \mu l} \varrho^{\mu l}+ \\
& +P^{\mu+1, l}\left(\frac{k}{2 h} B_{3}^{\mu l}-B_{1}^{\mu l}\right) P^{-1 \mu l-1} \varrho^{\mu l-1} .
\end{aligned}
$$

Using notations $2^{\circ}$ and $3^{\circ}$ we have

$$
\varrho^{\mu+1, l}=S_{\mu} \varrho^{\mu l}+k G_{\mu} \varrho^{\mu l}+k \varepsilon^{\mu l} .
$$

The proof of Lemma 2 is ended.

We introduce the notations:

$$
\begin{aligned}
\varrho^{\mu} & =\left(\ldots, \varrho^{\mu,-3}, \varrho^{\mu,-2}, \varrho^{\mu,-1}, \varrho^{\mu, 0}, \varrho^{\mu, 1}, \varrho^{\mu, 2}, \varrho^{\mu, 3}, \ldots\right), \\
S \varrho^{\mu} & =\left(\ldots, S_{\mu} \varrho^{\mu,-2}, S_{\mu} \varrho^{\mu,-1}, S_{\mu} \varrho^{\mu, 0}, S_{\mu} \varrho^{\mu, 1}, S_{\mu} \varrho^{\mu, 2}, \ldots\right), \\
G \varrho^{\mu} & =\left(\ldots, G_{\mu} \varrho^{\mu,-1}, G_{\mu} \varrho^{\mu, 0}, G_{\mu} \varrho^{\mu, 1}, G_{\mu} \varrho^{\mu, 2}, \ldots\right), \\
\epsilon^{\mu} & =\left(\ldots, \varepsilon^{\mu,-2}, \varepsilon^{\mu,-1}, \varepsilon^{\mu, 0}, \varepsilon^{\mu, 1}, \varepsilon^{\mu, 2}, \ldots\right) .
\end{aligned}
$$

We shall show the difference inequality which is satisfied by $\left\|\varrho^{\mu}\right\|$ and the estimations of the norms of the operators: $\left\|S \varrho^{\mu}\right\|,\left\|G \varrho^{\mu}\right\|,\left\|\varepsilon^{\mu}\right\|$.

LEMMA 3. The vectors $\varrho^{\mu}$ satisfy the difference equation

and

$$
\varrho^{\mu+i}=S \varrho^{\mu}+k G \varrho^{\mu}+k \varepsilon^{\mu}
$$

$$
\begin{aligned}
\left\|S \varrho^{\mu}\right\| & \leqslant(1+R k)\left\|\varrho^{\mu}\right\|, \\
\left\|G \varrho^{\mu}\right\| & \leqslant \frac{1}{h} R_{1}\left\|\varrho^{\mu}\right\|^{2}+R_{1}\left\|\varrho^{\mu}\right\|+O\left(k^{3 / 2}\right) . \\
\left\|\varepsilon^{\mu}\right\| & \leqslant O\left(k^{3 / 2}\right) .
\end{aligned}
$$

Proof. 1. The operators $S_{\mu}, G_{\mu}$ are defined at nodal points of the not belonging to the trapeze $T$ :

$$
T: 0 \leqslant t \leqslant \alpha, \frac{h}{k} t-a \leqslant x \leqslant-\frac{h}{k} t+a .
$$


We assume that the coefficients in the operators $G$ and $S$ outside the trapeze $T$ are constant with respect to $x$, i.e.

$$
\begin{array}{cc}
\Lambda(t, x)=\Lambda\left(t,-\frac{h}{k} t+a\right) & \text { for } x \geqslant-\frac{h}{k} t+a, \\
\Lambda(t, x)=\Lambda\left(t, \frac{h}{k} t-a\right) & \text { for } x \leqslant \frac{h}{k} t-\alpha .
\end{array}
$$

Hence it follows that, outside the trapeze $T_{1}, \varepsilon^{\mu l}$ are equal to zero:

$$
T_{1}: 0 \leqslant t \leqslant \alpha, \quad-\frac{h}{k} t-\alpha \leqslant x \leqslant \frac{h}{k} t+\alpha .
$$

Outside the trapeze $T_{1}$ all $\varrho^{\mu l}$ are zero. So $\varrho^{\mu l}$ belong to the set $\Omega$. The vectors in set $\Omega$ satisfy the relation

$$
\varrho^{\mu+1}=S \varrho^{\mu}+k G \varrho^{\mu}+k \varepsilon^{\mu} .
$$

2. At this point we shall estimate the norm $\left\|S \varrho^{\mu}\right\|$ in $\Omega$,

$$
\begin{aligned}
\left\|S \varrho^{\mu}\right\|^{2}= & \left(S \varrho^{\mu}, S \varrho^{\mu}\right) \\
& =\sum_{l=-\infty}^{\infty}\left[\varrho^{\mu l}+q^{\mu l}\left(\varrho^{\mu l+1}-\varrho^{\mu l-1}\right)+2 q^{2 \mu l}\left(\varrho^{\mu l+1}-2 \varrho^{\mu l}+\varrho^{\mu l-1}\right)\right]^{2},
\end{aligned}
$$

where $q^{\mu l}=\frac{k}{2 h} \Lambda^{\mu l}$,

$$
\begin{aligned}
& \left(S \varrho^{\mu}, S \varrho^{\mu}\right)=\sum_{l=-\infty}^{\infty}\left\{\varrho^{2 \mu l}+2 \varrho^{\mu l} q^{\mu l} \varrho^{\mu l+1}-2 \varrho^{\mu l} q^{\mu l} \varrho^{\mu l-1}+4 \varrho^{\mu l} q^{2 \mu l} \varrho^{\mu l+1}-\right. \\
& \quad-8 \varrho^{\mu l} q^{2 \mu l} \varrho^{\mu l}+4 \varrho^{\mu l} q^{2 \mu l} \varrho^{\mu l-1}+\left[q^{\mu l}\left(\varrho^{\mu l+1}-\varrho^{\mu l-1}\right)\right]^{2}+ \\
& \quad+\left[q^{\mu l}\left(\varrho^{\mu l+1}-2 \varrho^{\mu l}+\varrho^{\mu l-1}\right)\right]^{2}+4 q^{\mu l}\left(\varrho^{\mu l+1}-\varrho^{\mu l-1}\right) q^{2 \mu l}\left(\varrho^{\mu l+1}-2 \varrho^{\mu l}+\varrho^{\mu l-1}\right)+ \\
& \left.\quad+\left[2 q^{2 \mu l}\left(\varrho^{\mu l+1}-2 \varrho^{\mu l}+\varrho^{\mu l-1}\right)\right]^{2}-\left[q^{\mu l}\left(\varrho^{\mu l+1}-2 \varrho^{\mu l}+\varrho^{\mu l-1}\right)\right]^{2}\right\}
\end{aligned}
$$

The term composed of the last two components is negative as soon as $4 q^{4 \mu l}-q^{2 \mu l}<0$ (a matrix of negative components).

This can always be done by taking a sufficiently small proportion $k / h$. So after multiplying and reducing, we have

$$
\begin{aligned}
& \left(S \varrho^{\mu}, S \varrho^{\mu}\right) \leqslant \sum_{l=-\infty}^{\infty}\left\{\varrho^{2 \mu l}+2 \varrho^{\mu l} q^{\mu l} \varrho^{\mu l+1}-2 \varrho^{\mu l} q^{\mu l} \varrho^{\mu l-1}-4 \varrho^{\mu l} q^{2 \mu l} \varrho^{\mu l}+\right. \\
& \quad+2 \varrho^{\mu l+1} q^{2 \mu l} \varrho^{\mu l+1}+2 \varrho^{\mu l-1} q^{2 \mu l} \varrho^{\mu l-1}+4 \varrho^{\mu l+1} q^{3 \mu l} \varrho^{\mu l+1}-8 \varrho^{\mu l} q^{3 \mu l} \varrho^{\mu l+1}+ \\
& \left.\quad+8 \varrho^{\mu l-1} q^{3 \mu l} \varrho^{\mu l}-4 \varrho^{\mu l-1} q^{3 \mu l} \varrho^{\mu l-1}\right\} .
\end{aligned}
$$


From the regularity of values $\lambda_{i}$ follow the equalities

$$
\Lambda^{\mu l}=\Lambda^{\mu l \div 1}-\Lambda_{x}^{\mu l} h, \quad \Lambda^{\mu l}=\Lambda^{\mu l-1}+h \Lambda_{x}^{\mu l-1} .
$$

Since $q^{\mu l}=\frac{k}{2 h} \Lambda^{\mu l}$, so $q^{\mu l}=q^{\mu l+1}-q_{x}^{\mu l} h, q^{\mu l}=q^{\mu l-1}+h q_{x}^{\mu l-1}$.

After substitution in (14) multiplying and using the equalities

$$
\begin{aligned}
\sum_{l=-\infty}^{\infty} 2 \varrho^{\mu l} q^{\mu l} \varrho^{\mu l+1}-\sum_{l=-\infty}^{\infty} 2 \varrho^{\mu l} q^{\mu l-1} \varrho^{\mu l-1} & =0 \\
\sum_{l=-\infty}^{\infty} 2 \varrho^{\mu l+1} q^{2 \mu l+1} \varrho^{\mu l+1}+\sum_{l=-\infty}^{\infty} 2 \varrho^{\mu l-1} q^{2 \mu l-1} \varrho^{\mu l-1}-\sum_{l=-\infty}^{\infty} 4 \varrho^{\mu l} q^{2 \mu l} \varrho^{\mu l} & =0 \\
\sum_{l=-\infty}^{\infty} 4 \varrho^{\mu l+1} q^{3 \mu l+1} \varrho^{\mu l+1}-\sum_{l=-\infty}^{\infty} 4 \varrho^{\mu l-1} q^{3 \mu l-1} \varrho^{\mu l-1} & =0 \\
\sum_{l=-\infty}^{\infty} 8 \varrho^{\mu l} q^{3 \mu l} \varrho^{\mu l+1}-\sum_{l=-\infty}^{\infty} 8 \varrho^{\mu l-1} q^{3 \mu l-1} \varrho^{\mu l}=0 & =0
\end{aligned}
$$

we obtain

$$
\begin{aligned}
\left(S \varrho^{\mu},\right. & \left.S \varrho^{\mu}\right) \leqslant \sum_{l=-\infty}^{\infty}\left\{\varrho^{2 \mu l}-2 h \varrho^{\mu l} q_{x}^{\mu l-1} \varrho^{\mu l-1}-4 \varrho^{\mu l+1} q^{\mu l+1} q_{x}^{\mu l} \varrho^{\mu l+1} h+\right. \\
& +2 h^{2} \varrho^{\mu l+1} q_{x}^{2 \mu l} \varrho^{\mu l+1}+4 h \varrho^{\mu l-1} q^{\mu l-1} q_{x}^{\mu l-1} \varrho^{\mu l-1}+2 h^{2} \varrho^{\mu l-1} q_{x}^{2 \mu l-1} \varrho^{\mu l-1}+ \\
& +\varrho^{\mu l+1}\left[-12 q^{2 \mu l+1} q_{x}^{\mu l} h+12 q^{\mu l+1} q_{x}^{2 \mu l} h^{2}-4 h^{3} q_{x}^{3 \mu l}\right] \varrho^{\mu l+1}+ \\
& +\varrho^{\mu l-1}\left[-12 q^{2 \mu l-1} q_{x}^{\mu l-1} h-12 q^{\mu l-1} q_{x}^{2 \mu l-1} h^{2}-4 q_{x}^{3 \mu l-1} h^{3}\right] \varrho^{\mu l-1}+ \\
& \left.+\varrho^{\mu l-1}\left[24 q^{2 \mu l-1} q_{x}^{\mu l-1} h+24 q^{\mu l-1} q_{x}^{2 \mu l-1} h^{2}+8 q_{x}^{3 \mu l-1} h^{3}\right] \varrho^{\mu l}\right\} .
\end{aligned}
$$

Since

$\sum_{l=-\infty}^{\infty}\left\|\varrho^{\mu l+1}\right\|=\sum_{l=-\infty}^{\infty}\left\|\varrho^{\mu l}\right\|=\sum_{l=-\infty}^{\infty}\left\|\varrho^{\mu l-1}\right\| \quad$ and $\quad\left|\left(\varrho^{\mu l}, \varrho^{\mu l-1}\right)\right| \leqslant\left\|\varrho^{\mu l}\right\|^{2}+\left\|\varrho^{\mu l-1}\right\|^{2}$ and in virtue of the boundedness of the functions in assumption $Z$ we have

$$
\begin{aligned}
\left(S \varrho^{\mu}, S \varrho^{\mu}\right) \leqslant\left\|\varrho^{\mu}\right\|^{2}+\left(2 H+8 H^{2}\right. & \left.+48 H^{3}\right) k\left\|\varrho^{\mu}\right\|^{2}+ \\
& +\left(4 H^{2}+48 H^{3}\right) k^{2}\left\|\varrho^{\mu}\right\|^{2}+16 H^{3} k^{3}\left\|\varrho^{\mu}\right\|^{2} .
\end{aligned}
$$

From the inequalities $k<h<1$ we have

$$
\left(S \varrho^{\mu}, S \varrho^{\mu}\right) \leqslant\left\|\varrho^{\mu}\right\|^{2}+\left(2 H+12 H^{2}+112 H^{3}\right) k\left\|\varrho^{\mu}\right\|^{2} .
$$

Writing $R=2 H+12 H^{2}+112 H^{3}$, we have $\left(S \varrho^{\mu}, S \varrho^{\mu}\right) \leqslant(1+R k)\left\|\varrho^{\mu}\right\|^{2}$, 
i.e. $\left\|S \varrho^{\mu}\right\|^{2} \leqslant(1+R k)\left\|\varrho^{\mu}\right\|^{2}$, and hence

$$
\left\|S \varrho^{\mu}\right\| \leqslant(1+R k)\left\|\varrho^{\mu}\right\| .
$$

3. We know that $\varrho^{\mu+1}=S \varrho^{\mu}+k G \varrho^{\mu}+k \varepsilon^{\mu}$; hence

$$
\begin{aligned}
& \left\|\varrho^{\mu+1}\right\| \leqslant\left\|S \varrho^{\mu}\right\|+k\left\|G \varrho^{\mu}\right\|+k\left\|\varepsilon^{\mu}\right\|, \\
& \left\|\varrho^{\mu+1}\right\| \leqslant(1+R k)\left\|\varrho^{\mu}\right\|+k\left\|G \varrho^{\mu}\right\|+k\left\|\varepsilon^{\mu}\right\| .
\end{aligned}
$$

It is known that the operator $G_{\mu} \varrho^{\mu l}$ is a sum of operators:

$$
\begin{aligned}
& G_{\mu} \varrho^{\mu l}=E^{\mu l+1} \varrho^{\mu l+1}+E^{\mu l} \varrho^{\mu l}+E^{\mu l-1} \varrho^{\mu l-1}+P^{\mu+1, l} B_{u}^{\mu+1 / 2, l} P^{-1 \mu+1 / 2, l} \varrho^{\mu+1 / 2, l}+ \\
& +P^{\mu+1, l}\left(B_{1}^{\mu l}+\frac{k}{2 h} B_{2}^{\mu l}\right) P^{-1 \mu l+1} \varrho^{\mu l+1}-\frac{k}{2 h} P^{\mu+1, l}\left(B_{2}^{\mu l}+B_{3}^{\mu l}\right) P^{-1 \mu l} \varrho^{\mu l}+ \\
& +P^{\mu+1, l}\left(\frac{k}{2 h} B_{3}^{\mu l}-B_{1}^{\mu l}\right) P^{-1 \mu l-1} \varrho^{\mu l-1}+\frac{k}{2 h} P^{\mu+1, l} w_{1}^{\mu l}+P^{\mu+1, l} w_{2}^{\mu l}+\frac{k}{2 h} P^{\mu+1, l} w_{3}^{\mu l}
\end{aligned}
$$

Consequently the operator $G \varrho^{\mu}$ is a sum of operators. We shall estimate successively each term of the operator $G \varrho^{\mu}$ :

$$
\begin{aligned}
& \left\|E_{1} \varrho^{\mu}\right\|^{2}=\left(E_{1} \varrho^{\mu}, E_{1} \varrho^{\mu}\right)=\sum_{l=-\infty}^{\infty}\left(E^{\mu l+1} \varrho^{\mu l+1}, E^{\mu l+1} \varrho^{\mu l+1}\right)=\sum_{l=-\infty}^{\infty}\left\|E^{\mu l+1} \varrho^{\mu l+1}\right\|^{2} \\
& \leqslant \sum_{l=-\infty}^{\infty}\left\|E^{\mu l+1}\right\|^{2}\left\|\varrho^{\mu l+1}\right\|^{2} \leqslant\left(2, M^{2} H^{2}+4 H^{2} M\right)^{2} \sum_{l=-\infty}^{\infty}\left\|\varrho^{\mu l+1}\right\|^{2} \\
& =\left(2 H^{2} M^{2}+4 H^{2} M\right)^{2}\left\|\varrho^{\mu}\right\|^{2}, \\
& \text { (16) } \quad\left\|E_{1} \varrho^{\mu}\right\| \leqslant\left(2 H^{2} M^{2}+4 H^{2} M\right)\left\|\varrho^{\mu}\right\| \text {, } \\
& \left\|E^{0} \varrho^{\mu}\right\|=\left(\sum_{l=-\infty}^{\infty}\left\|E^{\mu l} \varrho^{\mu l}\right\|^{2}\right)^{1 / 2} \leqslant\left(H^{2}+2 H^{2} M^{2}+3 H^{2} M\right)\left\|\varrho^{\mu}\right\|, \\
& \text { (18) } \quad\left\|E_{-1} \varrho^{\mu}\right\|=\left(\sum_{l=-\infty}^{\infty}\left\|E^{\mu l-1} \varrho^{\mu l-1}\right\|^{2}\right)^{1 / 2} \leqslant\left(3 H^{2} M^{2}+4 H^{2} M\right)\left\|\varrho^{\mu}\right\| \text {. }
\end{aligned}
$$

We estimate the following term. From the above scheme we obtain

$$
\begin{aligned}
& r^{\mu+1 / 2, l}=r^{\mu l}+\frac{k}{2}\left[\left(A^{(u) \mu l}-A^{\mu l}\right) u_{x}^{\mu l}+\frac{k}{2} B_{u}^{\mu l} r^{\mu l}+\frac{k}{2} \eta^{\mu l}+\frac{k}{4 h} A^{\mu l}\left(r^{\mu l+1}-r^{\mu l-1}\right),\right. \\
& \begin{aligned}
& r^{\mu+1 / 2, l}= \frac{k}{4 h} A^{\mu l} r^{\mu l+1}+\left(I+\frac{k}{2} B_{u}^{\mu l}\right) r^{\mu l}+\frac{k}{2}\left[A^{(u) \mu l}-A^{\mu l}\right] u_{x}^{\mu l}- \\
&-\frac{k}{4 h} A^{\mu l} r^{\mu l-1}+\frac{k}{2} \eta^{\mu l}, \\
&\left\|r^{\mu+1 / 2}\right\| \leqslant M\left\|r^{\mu}\right\|+(1+M)\left\|r^{\mu}\right\|+M\left\|r^{\mu}\right\|+n c M\left\|r^{\mu}\right\|+O\left(k^{3 / 2}\right), \\
&\left\|r^{\mu+1 / 2}\right\| \leqslant(1+3 M+n c M)\left\|r^{\mu}\right\|+O\left(k^{3 / 2}\right), \\
& \text { (19) }
\end{aligned}
\end{aligned}
$$




\section{Hence}

(20)

$$
\begin{aligned}
& \left\|K_{1} \varrho^{\mu+1 / 2}\right\| \\
= & \left(\sum_{l=-\infty}^{\infty}\left(P^{\mu+1, l} B_{u}^{\mu+1 / 2, l} P^{-1 \mu+1 / 2, l} \varrho^{\mu+1 / 2, l}, P^{\mu+1, l} B_{u}^{\mu+1 / 2, l} P^{-1 \mu+1 / 2, l} \varrho^{\mu+1 / 2, l}\right)\right)^{1 / 2} \\
\leqslant & \left(B^{3} M+3 H^{3} M^{2}+n c H^{3} M^{2}\right)\left\|\varrho^{\mu}\right\|+O\left(k^{3 / 2}\right) .
\end{aligned}
$$

Now we estimate the operator $K_{2} \varrho^{\mu}=\left\{P^{\mu+1, l} B_{1}^{\mu l} P^{-1 \mu l+1} \varrho^{\mu l+1}\right\}$ :

$$
\begin{aligned}
\left\|K_{2} \varrho^{\mu}\right\|= & {\left[\sum_{-\infty}^{\infty}\left(P^{\mu+1, l} B_{1}^{\mu l} P^{-1 \mu l+1} \varrho^{\mu l+1}, P^{\mu+1, l} B_{1}^{\mu l} P^{-1 \mu l+1} \varrho^{\mu l+1}\right)\right]^{1 / 2} } \\
= & \left(\sum_{-\infty}^{\infty}\left\|P^{\mu+1, l} B_{1}^{\mu l} P^{-1 \mu l+1} \varrho^{\mu l+1}\right\|^{2}\right)^{3 / 2} \\
= & \left(\sum_{-\infty}^{\infty} \| \frac{1}{2 h} P^{\mu+1, l}\left[A^{\mu+1 / 2, l}-A^{(u) \mu+1 / 2, l}+\right.\right. \\
& \left.\left.+A^{(u) \mu+1 / 2, l}-A^{(u) \mu l}\right] P^{-1 \mu l+1} \varrho^{\mu, l+1} \|^{2}\right)^{1 / 2} \\
\leqslant & \left(H^{2} M+n c H^{2} M\right)\left\|\varrho^{\mu}\right\|+ \\
& +\left(\sum_{l=-\infty}^{\infty}\left\|\frac{1}{2 h} P^{\mu+1, l}\left(A^{\mu+1 / 2, l}-A^{(u) \mu+1 / 2, l}\right) P^{-1 \mu l+1} \varrho^{\mu l+1}\right\|^{2}\right)^{1 / 2} .
\end{aligned}
$$

Let us take the second component:

$$
\begin{aligned}
\left(\sum_{l=-\infty}^{\infty} \| \frac{1}{2 h}\right. & \left.P^{\mu+1, l}\left(A^{\mu+1 / 2, l}-A^{(u) \mu+1 / 2, l}\right) P^{-1 \mu, l+1} \varrho^{\mu l+1} \|^{2}\right)^{1 / 2} \\
& \leqslant\left(\sum_{-\infty}^{\infty} \frac{H^{4}}{4 h^{2}}\left\|\varrho^{\mu l+1}\right\|^{2}\left\|A^{\mu+1 / 2, l}-A^{(u) \mu+1 / 2, l}\right\|^{2}\right)^{1 / 2} \\
& \leqslant \frac{H^{2}}{2 h}\left(\sum_{-\infty}^{\infty}\left(\left\|\varrho^{\mu l+1}\right\|^{4}+\left\|A^{\mu+1 / 2, l}-A^{(u) \mu+1 / 2, l}\right\|^{4}\right)\right)^{1 / 2} \\
& \leqslant \frac{H^{2}}{2 h}\left(\sum_{-\infty}^{\infty}\left\|\varrho^{\mu l+1}\right\|^{2}+\sum_{l=-\infty}^{\infty}\left\|A^{\mu+1 / 2, l}-A^{(u) \mu+1 / 2, l}\right\|^{2}\right) \\
& =\frac{H^{2}}{2 h}\left\|\varrho^{\mu}\right\|^{2}+\frac{H^{2}}{2 h} \sum_{-\infty}^{\infty}\left\|A^{\mu+1 / 2, l}-A^{(u) \mu+1 / 2, l}\right\|^{2} .
\end{aligned}
$$


Since

$$
\frac{H^{2}}{2 h} \sum_{-\infty}^{\infty}\left\|A^{\mu+1 / 2, l}-A^{(u) \mu+1 / 2, l}\right\|^{2} \leqslant \frac{1}{2 h} n H^{2} M^{2}\left\|r^{\mu+1 / 2}\right\|^{2},
$$

using (19) we have

$$
\begin{gathered}
\frac{H^{2}}{2 h} \sum_{-\infty}^{\infty}\left\|A^{\mu+1 / 2, l}-A^{(u) \mu+1 / 2, l}\right\|^{2} \leqslant \frac{1}{2 h} n H^{2} M^{2}(H+3 H M+n c H M)^{2}\left\|\varrho^{\mu}\right\|^{2}+ \\
\quad+\frac{1}{h} n H^{2} M^{2}(H+3 H M+n c H M)\left\|\varrho^{\mu}\right\| O\left(k^{3 / 2}\right)+\frac{1}{2 h} n H^{2} M^{2} O\left(k^{3 / 2}\right) .
\end{gathered}
$$

Finally we have

$$
\begin{aligned}
& \left\|K_{2} \varrho^{\mu}\right\| \leqslant\left[H^{2}+n H^{2} M^{2}(H+3 H M+n c H M)^{2}\right] \frac{1}{2 h}\left\|\varrho^{\mu}\right\|^{2}+ \\
& \quad+\left[H^{2} M+n c H^{2} M+n H^{2} M^{2}(H+3 H M+n c H M)\right]\left\|\varrho^{\mu}\right\|+O\left(k^{2}\right) .
\end{aligned}
$$

We estimate the operator $K_{3} \varrho^{\mu}=\left\{P^{\mu+1, l} B_{2}^{\mu l} P^{-1 \mu l+1} \varrho^{\mu l+1}\right\}$,

$$
\begin{aligned}
& \left\|K_{\mathrm{B}} \varrho^{\mu}\right\|=\left(\sum_{-\infty}^{\infty}\left\|P^{\mu+1, l} B_{2}^{\mu l} P^{-1 \mu l+1} \varrho^{\mu l+1}\right\|^{2}\right)^{1 / 2} \\
\leqslant & \left(\sum_{-\infty}^{\infty}\left\|\frac{1}{h} P^{\mu+1, l}\left(A^{(u) \mu+1 / 2, l} A^{(u) \mu l+1 / 2}-A^{(u) \mu l} A^{(u) \mu l}\right) P^{-1 \mu l+1} \varrho^{\mu l+1} \cdot\right\| \|^{2}+\right. \\
& +\left(\sum_{-\infty}^{\infty / 2}\left\|\frac{1}{h} P^{\mu+1, l}\left(A^{\mu+1 / 2, l} A^{\mu, l+1 / 2}-A^{(u) \mu+1 / 2, l} A^{(u) \mu, l+1 / 2}\right) P^{-1 \mu l+1} \varrho^{\mu l+1}\right\|^{2}\right)^{1 / 2} \\
\leqslant & \left(2 H^{2} M^{2}+2 n c H^{2} M^{2}\right)\left\|\varrho^{\mu}\right\|+ \\
& +\left(\sum_{-\infty}^{\infty}\left\|\frac{1}{h} P^{\mu+1, l}\left(A^{\mu+1 / 2, l} A^{\mu l+1 / 2}-A^{(u) \mu+1 / 2, l} A^{(u) \mu l+1 / 2}\right) P^{-1 \mu l+1} \varrho^{\mu l+1}\right\|^{2}\right)^{1 / 2}
\end{aligned}
$$

We shall estimate the last term:

$$
\begin{aligned}
& \left(\sum_{-\infty}^{\infty}\left\|\frac{1}{h} P^{\mu+1, l}\left(A^{\mu+1 / 2, l} A^{\mu l+1 / 2}-A^{(u) \mu+1 / 2, l} A^{(u) \mu l+1 / 2}\right) P^{-1 \mu l+1} \varrho^{\mu l+1}\right\|^{2}\right)^{1 / 2} \\
& \leqslant\left(\sum_{-\infty}^{\infty}\left\|\frac{1}{h} P^{\mu+1, l}\left(A^{\mu+1 / 2, l} A^{\mu l+1 / 2}-A^{(u) \mu+1 / 2, l} A^{\mu, l+1 / 2}\right) P^{-1 \mu l+1} \varrho^{\mu l+1}\right\|\right)^{2}+
\end{aligned}
$$




$$
\begin{aligned}
& +\left(\sum_{-\infty}^{\infty}\left\|\frac{1}{h} P^{\mu+1, l}\left(A^{(u) \mu+1 / 2, l} A^{\mu l+1 / 2}-A^{(u) \mu+1 / 2, l} A^{(u) \mu l+1 / 2}\right) P^{-1 \mu l+1} \varrho^{\mu l+1}\right\|^{2}\right)^{1 / 2} \\
\leqslant & \frac{1}{h} n H^{3} M^{2}\left\|\varrho^{\mu}\right\|^{2}+\frac{1}{h} H^{2} M\left(\sum_{-\infty}^{\infty}\left\|A^{\mu+1 / 2, l}-A^{(u) \mu+1 / 2, l}\right\|^{2}\left\|\varrho^{\mu l+1}\right\|^{2}\right)^{1 / 2} \\
\leqslant & \frac{1}{h} n H^{3} M^{2}\left\|\varrho^{\mu}\right\|^{2}+\frac{1}{h} H^{2} M^{3}\left(\sum_{-\infty}^{\infty}\left\|r^{\mu+1 / 2, l}\right\|^{2}\left\|\varrho^{\mu l+1}\right\|^{2}\right)^{1 / 2} \\
\leqslant & \frac{1}{h} n H^{3} M^{2}\left\|\varrho^{\mu}\right\|^{2}+\frac{1}{h} H^{2} M^{3}\left(\sum_{-\infty}^{\infty}\left[\left\|r^{\mu+1 / 2, l}\right\|^{4}+\left\|\varrho^{\mu l+1}\right\|^{4}\right]\right)^{1 / 2} \\
\leqslant & \frac{1}{h} n H^{3} M^{2}\left\|\varrho^{\mu}\right\|^{2}+\frac{1}{h} H^{2} M^{3}\left(\left\|r^{\mu+1 / 2}\right\|^{2}+\left\|\varrho^{\mu}\right\|^{2}\right) \\
\leqslant & \frac{1}{h}\left[n H^{3} M^{2}+H^{2} M^{3}+H^{2} M^{3}(H+3 H M+n c H M)^{2}\right]\left\|\varrho^{\mu}\right\|^{2}+ \\
& +2 H^{2} M^{3}(H+3 H M+n c H M)\left\|\varrho^{\mu}\right\|+O\left(k^{2}\right) .
\end{aligned}
$$

Using this inequality, we finally have

(22) $\quad\left\|K_{3} \varrho^{\mu}\right\| \leqslant \frac{1}{h}\left[n H^{3} M^{2}+H^{2} M^{3}+H^{2} M^{3}(H+3 H M+n c H M)^{2}\right]\left\|\varrho^{\mu}\right\|^{2}+$$$
+\left[2 H^{2} M^{2}+2 n c H^{2} M^{2}+2 H^{3} M^{3}+6 H^{3} M^{4}+2 n c H^{3} M^{4}\right]\left\|\varrho^{\mu}\right\|+O\left(k^{2}\right) .
$$

The norm of the operator $K_{4} \varrho^{\mu}=\left\{P^{\mu+1, l} B_{2}^{\mu l} P^{-1 \mu l} \varrho^{\mu l}\right\}$ is estimated in the same way as the norm of the operator $K_{8} \varrho^{\mu}$ and an identical result is obtained

$$
\begin{aligned}
& \left\|K_{4} \varrho^{\mu}\right\| \leqslant\left[n H^{3} M^{2}+H^{2} M^{3}+H^{2} M^{3}(H+3 H M+n c H M)^{2}\right] \frac{1}{h}\left\|\varrho^{\mu}\right\|^{2}+ \\
+ & {\left[2 H^{2} M^{2}+2 n c H^{2} M^{2}+2 H^{3} M^{3}+6 H^{3} M^{4}+2 n c H^{3} M^{4}\right]\left\|\varrho^{\mu}\right\|+O\left(k^{2}\right) . }
\end{aligned}
$$

The norm of the operator $K_{5} \varrho^{\mu}=\left\{P^{\mu+1, l} B_{3}^{\mu l} P^{-1 \mu l} \varrho^{\mu l}\right\}$ is estimated by the same term as $\left\|K_{4} \varrho^{\mu}\right\|$ and $\left\|K_{3} \varrho^{\mu}\right\|$.

Analogically we estimate the norm of the operator

$$
K_{6} \varrho^{\mu}=\left\{P^{\mu+1, l}\left(\frac{k}{2 h} B_{3}^{\mu l}-B_{1}^{\mu l}\right) P^{-1 \mu l-1} \varrho^{\mu l-1}\right\}
$$

(24) $\left\|K_{6} \varrho^{\mu}\right\| \leqslant \frac{1}{h}\left[H^{2}+n H^{3} M^{2}+H^{2} M^{3}+\left(H^{2} M^{3}+n H^{2} M^{2}\right)(H+3 H M+\right.$

$$
\begin{aligned}
& \left.+n c H M)^{2}\right]\left\|\varrho^{\mu}\right\|^{2}+\left[H^{2} M+2 H^{2} M^{2}+n c H^{2} M+2 n c H^{2} M^{2}+2 H^{3} M^{3}+\right. \\
& \left.+6 H^{3} M^{4}+2 n c H^{3} M^{4}+n H^{3} M^{2}+3 n H^{3} M^{3}+n^{2} c H^{3} M^{3}\right]\left\|\varrho^{\mu}\right\|+O\left(k^{2}\right) .
\end{aligned}
$$


4. There are three vectors to estimate:

$$
w_{1}^{\mu}=\left\{P^{\mu+1, l} w_{1}^{\mu l}\right\}, \quad w_{2}^{\mu}=\left\{P^{\mu+1, l} w_{2}^{\mu l}\right\}, \quad w_{3}^{\mu}=\left\{P^{\mu+1, l} w_{3}^{\mu l}\right\},
$$

(25) $\quad\left\|w_{1}^{\mu}\right\| \leqslant H\left(\sum_{-\infty}^{\infty}\left\|A^{(u) \mu+1 / 2, l} A^{(u) \mu, l+1 / 2}-A^{\mu+1 / 2, l} A^{\mu l+1 / 2}\right\|^{2}\left\|u_{x}^{\mu l}\right\|^{2}\right)^{1 / 2}+$

$$
\begin{aligned}
& +H\left(\sum_{-\infty}^{\infty}\left\|A^{(u) \mu+1 / 2, l} A^{(u) \mu l-1 / 2}-A^{\mu+1 / 2, l} A^{\mu l-1 / 2}\right\|^{2}\left\|u_{x}^{\mu l}\right\|^{2}\right)^{1 / 2} \\
\leqslant & n c H\left(\sum_{-\infty}^{\infty}\left\|A^{(u) \mu+1 / 2, l} A^{(u) \mu l+1 / 2}-A^{\mu+1 / 2, l} A^{\mu l+1 / 2}\right\|^{2}\right)^{1 / 2}+ \\
& +n c H\left(\sum_{-\infty}^{\infty}\left\|A^{(u) \mu+1 / 2, l} A^{(u) \mu l-1 / 2}-A^{\mu+1 / 2, l} A^{\mu l-1 / 2}\right\|^{2}\right)^{1 / 2} \\
\leqslant & n^{2} c H\left[M\left\|r^{\mu+1 / 2}\right\|+M\left\|r^{\mu}\right\|\right]+n^{2} c H\left[M\left\|r^{\mu+1 / 2}\right\|+M\left\|r^{\mu}\right\|\right] \\
\leqslant & \left(4 n^{2} c B^{2} M+6 n^{2} c H^{2} M^{2}+2 n^{3} c^{2} H^{2} M^{2}\right)\left\|\varrho^{\mu}\right\|+2 n^{2} c H M O\left(k^{3 / 2}\right)
\end{aligned}
$$

(26) $\left\|w_{2}^{\mu}\right\| \leqslant\left(n^{2} c H^{2} M+3 n^{2} c H^{2} M^{2}+n^{3} c^{2} H^{2} M^{2}\right)\left\|\varrho^{\mu}\right\|+n^{2} c H M O\left(k^{3 / 2}\right)$,

(27) $\left\|w_{3}^{\mu}\right\| \leqslant\left(2 n H^{2} M^{2}+4 n H^{2} M^{3}+2 n^{2} c H^{2} M^{3}+2 H^{2} M^{2}\right)\left\|\varrho^{\mu}\right\|+$

$$
+2 n H M^{2} O\left(k^{3 / 2}\right),
$$

(28) $\left\|\varepsilon^{\mu}\right\| \leqslant\left(\sum_{-\infty}^{\infty}\left\|\varepsilon^{\mu l}\right\|^{2}\right)^{1 / 2} \leqslant\left(\sum_{-\infty}^{\infty} O\left(k^{4}\right)\right)^{1 / 2} \leqslant\left(O\left(k^{3}\right)\right)^{1 / 2}=O\left(k^{3 / 2}\right)$.

Considering all these inequality, we obtain an estimate for $\left\|G \varrho^{\mu}\right\|$,

$$
\left\|G \varrho^{\mu}\right\| \leqslant \frac{1}{h} R_{1}\left\|\varrho^{\mu}\right\|^{2}+R_{1}\left\|\varrho^{\mu}\right\|+O\left(k^{3 / 2}\right),
$$

where

$$
\begin{aligned}
R_{1}= & \max \left[a_{1}, a_{2}\right], \\
a_{1}= & 2 H^{2}+4 n H^{3} M^{2}+4 H^{2} M^{3}+\left(2 n H^{2} M^{2}+4 H^{2} M^{3}\right)(H+3 H M+n c H M)^{2}, \\
a_{2}= & H^{2}+13 H^{2} M+17 H^{2} M^{2}+H^{3} M+3 H^{3} M^{2}+n c H^{3} M^{2}+2 n c H^{2} M+ \\
& +2 n H^{3} M^{2}+6 n H^{3} M^{3}+2 n^{2} c H^{3} M^{3}+8 n c H^{2} M^{2}+8 H^{3} M^{3}+ \\
& +24 H^{3} M^{4}+8 n c H^{3} M^{4}+5 n^{2} c H^{3} M^{4}+9 n^{2} c H^{2} M^{2}+2 n^{3} c H^{2} M^{2}+ \\
& +n^{3} c^{2} H^{2} M^{2}+2 n H^{2} M^{2}+4 n H^{2} M^{3}+2 n^{2} c H^{2} M^{3} .
\end{aligned}
$$

The proof of Lemma 3 is ended. 
4. Proof of Theorem 1. Considering (13), (15), (28), (29) we have

$$
\frac{\left\|\varrho^{\mu+1}\right\|-\left\|\varrho^{\mu}\right\|}{k} \leqslant \frac{1}{h} R_{1}\left\|\varrho^{\mu}\right\|^{2}+\left(R_{1}+R\right)\left\|\varrho^{\mu}\right\|+O\left(k^{3 / 2}\right) .
$$

$O\left(k^{3 / 2}\right)=O\left(h^{3 / 2}\right)$ because $h / k$ is constant.

Let us denote $R_{1}+R=L$; then we have

$$
\begin{aligned}
& \frac{\left\|\varrho^{\mu+1}\right\|-\left\|\varrho^{\mu}\right\|}{k} \leqslant L h^{-3 / 2}\left\|\varrho^{\mu}\right\|^{2}+L\left\|\varrho^{\mu}\right\|+O\left(h^{3 / 2}\right), \\
& \frac{\left\|\varrho^{\mu+1}\right\|-\left\|\varrho^{\mu}\right\|}{k} \leqslant L h^{-3 / 2}\left[\left\|\varrho^{\mu}\right\|^{2}+2 h^{3 / 2}\left\|\varrho^{\mu}\right\|+h^{3}\right], \\
& \frac{\left\|\varrho^{\mu+1}\right\|-\left\|\varrho^{\mu}\right\|}{k} \leqslant L h^{-3 / 2}\left[\left\|\varrho^{\mu}\right\|+h^{3 / 2}\right]^{2} .
\end{aligned}
$$

From the theorem on difference inequalities we have

$$
\left\|\varrho^{\mu}\right\| \leqslant L \mu \hbar h^{3 / 2} /(1-L \mu \hbar),
$$

cf. the initial conditions $\left\|\varrho^{0}\right\|=\mathbf{0}$.

Since $1-L \mu k>0$, we have $\mu k<1 / L$.

We have

$$
\left\|r^{\mu l}\right\|=\left\|P^{-1 \mu l} \varrho^{\mu l}\right\| \leqslant\left\|P^{-1 \mu l}\right\|\left\|\varrho^{\mu l}\right\|=H\left\|\varrho^{\mu l}\right\| \leqslant H\left\|\varrho^{\mu}\right\| \leqslant \frac{H L \mu k h^{3 / 2}}{1-L \mu k} .
$$

The proof of the theorem is ended. 